\title{
Efficacy and tolerability of methotrexate therapy for refractory intestinal Behçet's disease: a single center experience
}

\author{
Jihye Park ${ }^{1}$, Jae Hee Cheon ${ }^{1,2}$, Yehyun Park ${ }^{1,2}$, Soo Jung Park ${ }^{1,2}$, Tae Il Kim ${ }^{1,2}$, Won Ho Kim ${ }^{1,2}$ \\ ${ }^{1}$ Department of Internal Medicine and ${ }^{2}$ Institute of Gastroenterology, Yonsei University College of Medicine, Seoul, Korea
}

The conventional treatments for intestinal Behçet's disease (BD) include 5-aminosalicylic acids (5-ASAs), systemic corticosteroids, and immunosuppressive agents such as thiopurines. However, long-term use of corticosteroids is associated with steroid dependency and resistance, requiring additional immunosuppressant therapy or surgery. Azathioprine and 6-mercaptopurine (6-MP) are associated with bone marrow suppression and reported to occur in $56.4 \%$ after treatment with the maximal doses of azathioprine and 6-MP in Korean patients with IBD, which was a considerably higher incidence than that reported in Western studies. ${ }^{1}$ Furthermore, BD may be associated with myelodysplastic syndrome, which might hamper the use of thiopurines in such patients. As intestinal BD is often refractory to these agents, the use of anti-tumor necrosis factor- $\alpha$ (anti-TNF- $\alpha$ ) agents such as infliximab and adalimumab has recently been encouraged for the management of intestinal BD. A modest increase in the incidence of serious infection with biologics has been observed in methodologically rigorous studies. ${ }^{2}$

Methotrexate (MTX), an analogue of folic acid and aminopterin, has antineoplastic function at high doses and inhibits lymphocyte proliferation and pro-inflammatory cytokines at low doses. MTX is currently being increasingly used in combination with anti-TNF- $\alpha$ agents to prevent immunogenicity in patients with CD. To our knowledge, few

Received October 11, 2017. Revised November 13, 2017.

Accepted November 17, 2017.

Correspondence to Jae Hee Cheon, Department of Internal Medicine,

Yonsei University College of Medicine, 50-1 Yonsei-ro, Seodaemun-gu,

Seoul 03722, Korea. Tel: +82-2-2228-1990, Fax: +82-2-393-6884, E-mail:

Geniushee@yuhs.ac studies have evaluated the efficacy of MTX in patients with intestinal BD. In this study, we aimed to investigate the effect and tolerability of MTX monotherapy and the combination MTX with adalimumab for patients with refractory intestinal BD.

Between March 2005 and February 2017, a total of 606 patients with intestinal BD were registered at the IBD Clinic of Severance Hospital, Seoul, Korea. Patients are diagnosed with intestinal BD if gastrointestinal symptoms are present, and typical ulcerative lesions are documented by objective measures. ${ }^{3}$ Patients with refractory intestinal BD were defined as follows: (1) those with active intestinal ulceration based on colonoscopy despite intensive therapy with 5 -ASAs, corticosteroids, azathioprine, or anti-TNF- $\alpha$ agents for $>8$ weeks, and (2) those with steroid dependence. Ten patients were treated with MTX for refractory intestinal BD. The patients received a variety of doses of MTX, which were given either through SC injection or orally on a weekly basis at the discretion of physicians. Adalimumab was administered at a dose of $160 \mathrm{mg}$ in week $0,80 \mathrm{mg}$ in week 2 , and 40 mg every other week for patients with refractory intestinal BD. The primary outcome of interest was corticosteroid-free remission at 3 and 6 months for refractory intestinal BD. The secondary outcomes of interest were disease activity index for the intestinal Behçet's disease (DAIBD) score, CRP level, and ESR levels at 3 and 6 months. ${ }^{4}$ Comparisons were made using a Wilcoxon signed rank test. All statistical analyses were assessed with the SPSS version 23.0 (IBM Corp., Armonk, NY, USA). A $P$-value of $<0.05$ was considered statistically significant.

Ten patients were treated with MTX for active refractory

๑ Copyright 2018. Korean Association for the Study of Intestinal Diseases. All rights reserved.

This is an Open Access article distributed under the terms of the Creative Commons Attribution Non-Commercial License (http://creativecommons.org/licenses/by-nc/4.0)

which permits unrestricted non-commercial use, distribution, and reproduction in any medium, provided the original work is properly cited. 
intestinal $\mathrm{BD}$. The mean age was $52.9 \pm 9.6$ years (median, 48.5 years; range, $41-70$ years) and the mean disease duration was $7.2 \pm 5.9$ years (median, 7.0 years; range, $1-18$ years) (Table 1). Four patients received MTX as a monotherapy and 6 patients received MTX with adalimumab. The indica-

Table 1. Baseline Characteristics of the Study Patients

\begin{tabular}{|c|c|}
\hline Variable & Value $(n=10)$ \\
\hline Age (yr) & $52.9 \pm 9.6$ \\
\hline Male sex & $5(50.0)$ \\
\hline Time since diagnosis (yr) & $7.2 \pm 5.9$ \\
\hline \multicolumn{2}{|l|}{ Ulcer location } \\
\hline Terminal ileum & $2(20.0)$ \\
\hline IC valve & $5(50.0)$ \\
\hline Colon & $1(10.0)$ \\
\hline Anastomosis site & $2(20.0)$ \\
\hline Deep ulcer (vs. shallow ulcer) & $6(60.0)$ \\
\hline Volcano ulcer (vs. oval ulcer) & $3(30.0)$ \\
\hline \multicolumn{2}{|l|}{ Ulcer size (cm) } \\
\hline $1-2$ & $4(40.0)$ \\
\hline $2-3$ & $1(10.0)$ \\
\hline$\geq 3$ & $5(50.0)$ \\
\hline No. of surgery & $1.4 \pm 2.3$ \\
\hline No. of perforation & $0.8 \pm 1.0$ \\
\hline \multicolumn{2}{|l|}{ Extraintestinal manifestation } \\
\hline Oral ulceration & $10(100.0)$ \\
\hline Genital ulceration & $4(40.0)$ \\
\hline Uveitis & $1(10.0)$ \\
\hline Skin lesion & $6(60.0)$ \\
\hline Arthritis & $7(70.0)$ \\
\hline Nervous system & 0 \\
\hline Vascular system & 0 \\
\hline \multicolumn{2}{|l|}{ Previous medication } \\
\hline Thiopurine & $7(70.0)$ \\
\hline \multicolumn{2}{|l|}{ Anti-TNF- $\alpha$} \\
\hline Infliximab & $3(30.0)$ \\
\hline Adalimumab & $2(20.0)$ \\
\hline Infliximab, adalimumab & $1(10.0)$ \\
\hline DAIBD total score & $82.5 \pm 36.8$ \\
\hline CRP level (mg/L) & $57.8 \pm 36.0$ \\
\hline ESR level (mm/hr) & $38.0 \pm 36.1$ \\
\hline
\end{tabular}

Values are presented as mean \pm SD or number (\%).

IC, ileocecal; TNF, tumor necrosis factor; DAIBD, disease activity index for intestinal Behçet's disease. tions for MTX therapy were as follows: thiopurine intolerance, $10 \%$; failure of anti-TNF- $\alpha$ agents, $60 \%$; and corticosteroid dependence, $30 \%$ of patients. The administration route was oral in 8 patients, and SC or intramuscular (IM) route in 2 patients. The mean MTX dose for refractory intestinal BD was $13.0 \mathrm{mg}$ (median, $12.5 \mathrm{mg}$; range, $7.5-20.0 \mathrm{mg}$ ) (Table 2). The mean maintenance duration was $7.9 \pm 5.0$ months (median, 6.5 months; range, 3-20 months). The reasons for discontinuing MTX were clinical inefficiency in 3 patients and self-interruption in 1 patient. One patient underwent major surgery while on MTX and 4 patients required hospitalization because of severe abdominal pain.

Table 2. Details of Methotrexate Drug Use

\begin{tabular}{|c|c|}
\hline Variable & Value $(n=10)$ \\
\hline Age at initiation of MTX (yr) & $52.3 \pm 9.7$ \\
\hline Disease duration at initiation of MTX (yr) & $6.7 \pm 5.5$ \\
\hline \multicolumn{2}{|l|}{ Initial administration route } \\
\hline Parenteral & $2(20.0)$ \\
\hline Oral & $8(80.0)$ \\
\hline MTX dose (mg/wk) & $13.0 \pm 3.3$ \\
\hline \multicolumn{2}{|l|}{ MTX regimen } \\
\hline MTX monotherapy & $4(40.0)$ \\
\hline MTX with anti-TNF- $\alpha$ & $6(60.0)$ \\
\hline \multicolumn{2}{|l|}{ Concomitant medication } \\
\hline $5-A S A$ & $9(90.0)$ \\
\hline Colchicine & $4(40.0)$ \\
\hline Corticosteroid & $9(90.0)$ \\
\hline \multicolumn{2}{|l|}{ Anti-TNF- $\alpha$} \\
\hline Infliximab & 0 \\
\hline Adalimumab & $6(60.0)$ \\
\hline Opioid & $3(30.0)$ \\
\hline NSAID & $6(60.0)$ \\
\hline Antibiotics & $4(40.0)$ \\
\hline \multicolumn{2}{|l|}{ Indication for MTX } \\
\hline Thiopurine intolerance & $1(10.0)$ \\
\hline Thiopurine ineffectiveness & 0 \\
\hline Failure of monotherapy anti-TNF- $\alpha$ & $6(60.0)$ \\
\hline Corticosteroid dependence & $3(30.0)$ \\
\hline \multicolumn{2}{|l|}{ Reason discontinue $(n=4)$} \\
\hline Clinical ineffectiveness & $3(75.0)$ \\
\hline Self-interruption & $1(25.0)$ \\
\hline
\end{tabular}

Values are presented as mean \pm SD or number (\%).

MTX, methotrexate; TNF, tumor necrosis factor; ASA, aminosalicylic acid. 
Of the 10 patients receiving MTX, 3 patients (30\%) achieved steroid-free remission at 3 months. Moreover, of the 8 patients receiving MTX for $>6$ months, 4 patients $(50 \%)$ achieved steroid-free remission at 6 months. The mean DAIBD score was $82.5 \pm 36.8$ at baseline. This decreased to $72.5 \pm 40.7$ at 3 months and to $68.8 \pm 51.5$ at 6 months; however, there was no significant difference $(P=0.309)$. The mean CRP level was $38.0 \pm 36.1 \mathrm{mg} / \mathrm{L}$ at baseline. This decreased to $16.1 \pm 28.5 \mathrm{mg} / \mathrm{L}$ at 3 months and to $6.7 \pm 7.2 \mathrm{mg} / \mathrm{L}$ at 6 months, showing a statistical significance between 0 and 6 months $(P=0.039)$ (Fig. 1A). The mean ESR level was 57.8 \pm 36.0 $\mathrm{mm} / \mathrm{hr}$ at baseline. This decreased to $42.8 \pm 36.8 \mathrm{~mm} / \mathrm{hr}$ at 3 months and to $30.4 \pm 28.5 \mathrm{~mm} / \mathrm{hr}$ at 6 months, showing no significant difference $(P=0.078)$ (Fig. 1B). Eight patients were administered MTX after 6 months, and 2 patients (25.0\%) presented a relapse during this treatment. One patient underwent major surgery (right hemicolectomy) due to bowel perforation during MTX combination therapy and the other 1 patient underwent admission to control abdominal pain during MTX monotherapy. No serious adverse events were observed during 6 months of treatment. Nausea was reported by $20 \%$ of the patients.

Our study is the first to demonstrate the efficacy and tolerability of MTX monotherapy and MTX and adalimumab combination therapy. Three patients (30\%) at 3 months and 4 patients $(50 \%)$ at 6 months responded to MTX, achieving steroid-free remission. Furthermore, the serum CRP level was significantly decreased at 6 months compared with the baseline. The serum ESR and DAIBD score tended to decrease; however, there were no statistically significant

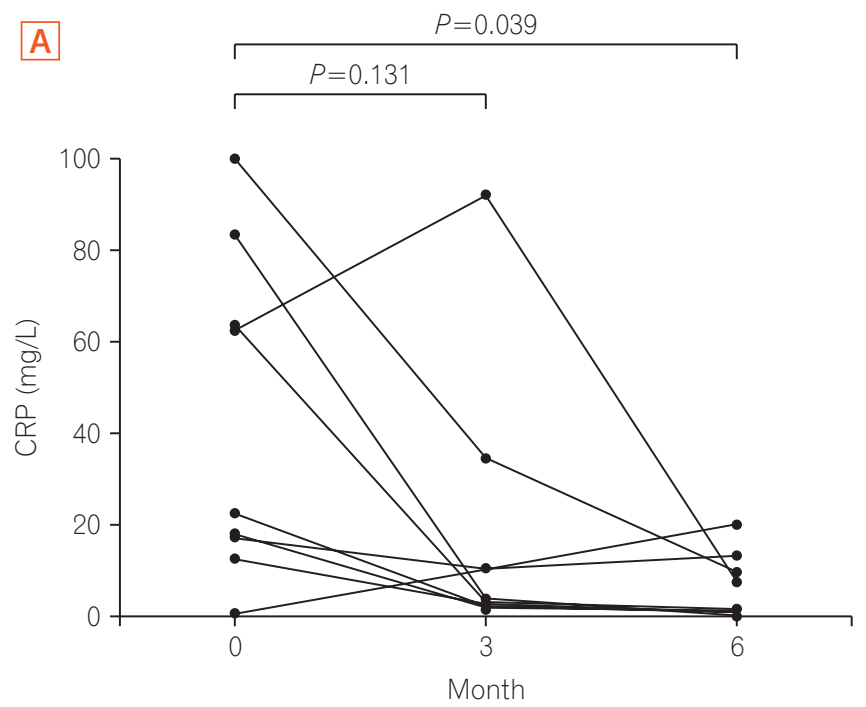

differences. In 1987, a dose of $25 \mathrm{mg}$ MTX was first used for patients with IBD, administered through the IM route for 12 weeks and then switched to a $7.5 \mathrm{mg}$ oral formulation. Kozarek et al. ${ }^{5}$ reported that in 16 of 31 patients with IBD, the corticosteroid dose could be tapered considerably. In a recent Cochrane review, IM MTX had a significant effect in patients with CD. Ardizzone et al. ${ }^{6}$ reported that $25 \mathrm{mg}$ / week MTX is effective in inducing remission in patients with chronic active CD, with a therapeutic efficacy being comparable to that of $2 \mathrm{mg} / \mathrm{kg} /$ day azathioprine after 3 months (44\% vs. $33 \%, P=0.28$ ) and 6 months ( $56 \%$ vs. $63 \%, P=0.39$ ). Moreover, Mate-Jimenez et al. suggested that $1.5 \mathrm{mg} / \mathrm{kg} /$ day 6-MP or $15 \mathrm{mg} /$ week MTX added to prednisolone could be effective in maintaining remission in patients with steroiddependent CD (53.3\% vs. $66.6 \%, P<0.001) .{ }^{7}$ Both trials had a small size; however, they showed a similar efficacy of MTX to that of thiopurines. The advantage with the use of MTX is that cancer incidence is lower than that with azathioprine and the frequency of bone marrow suppression is low. MTX might be superior to azathioprine because intestinal BD is often accompanied by myelodysplastic syndrome with bone marrow dysfunction. ${ }^{8}$ Furthermore, MTX is currently being increasingly used in combination with anti-TNF- $\alpha$ agents for patients with IBD to prevent immunogenicity. In COMMIT (Combination of Maintenance of Methotrexate-Infliximab Trial), patients with CD who received MTX were found to be less likely to develop antibodies to infliximab than those who received infliximab alone ( $4 \% \mathrm{vs} .20 \%, P=0.01){ }^{9}$

This study has several limitations. First, it was a retrospective, cross-sectional, and small study that was based

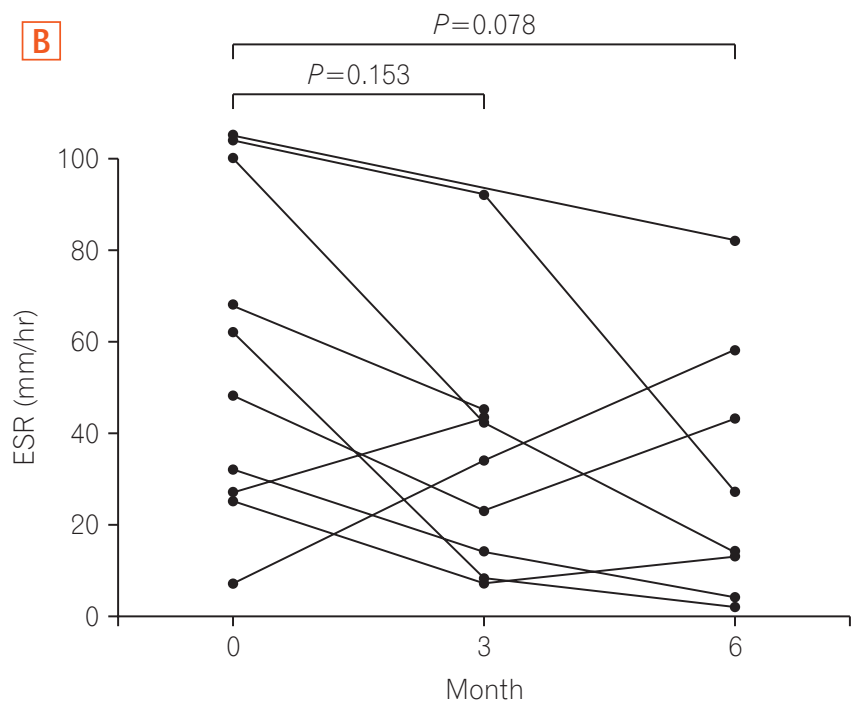

Fig. 1. (A) Serial changes in CRP level and (B) ESR at 3 and 6 months after the initiation of methotrexate treatment in patients with refractory intestinal Behçet's disease. 
on a medical record review. We could not draw a concrete conclusion that MTX monotherapy is effective in refractory intestinal BD because the number of patients is not enough. Nevertheless, we could report the possibility of MTX for patients with refractory BD by analyzing a well-organized, large database containing laboratory data and disease severity score index. Second, data were collected from a single tertiary hospital, which might limit the generalization of our findings to the general population. However, our center treats the greatest pool of patients with BD in Korea, and as patients with intestinal BD mostly visit tertiary hospitals, we believe that the bias was minimal.

MTX is an effective drug for inducing and maintaining remission in CD, and it is an efficient drug both as a monotherapy and a combination therapy with an anti-TNF- $\alpha$ agent (to prevent immunization). The use of MTX in intestinal BD has not yet been established because of insufficient evidence. On the basis of our study, MTX might be an effective treatment for refractory intestinal BD. Further larger studies would be necessary to validate our conclusion.

\section{FINANCIAL SUPPORT}

This research was supported by a grant (A120176) from the Korean Health Technology R\&D Project through the Korea Health Industry Development Institute, which is funded by the Ministry of Health and Welfare, Republic of Korea; and a grant (NRF-2017R1A2B4001848) from the Basic Science Research Program through the National Research Foundation of Korea, which is funded by the Ministry of Science, ICT and Future Planning.

\section{CONFLICT OF INTEREST}

No potential conflict of interest relevant to this article was reported.

\section{AUTHOR CONTRIBUTION}

Guarantor of the article: J.H.C.

Specific author contributions: J.P., acquisition of data; analysis and interpretation of data; drafting of the manuscript. Y.P., study concept and design; critical revision of the manuscript for important intellectual content. S.J.P., study concept and design; critical revision of the manuscript for important intellectual content. T.I. K., study concept and design; critical revision of the manuscript for important intellectual content. W.H.K., study concept and design; critical revision of the manuscript for important intellectual content. J.H.C., acquisition of data; study concept and design; critical revision of the manuscript for important intellectual content. All authors approved the final version of the article, including the authorship list.

\section{REFERENCES}

1. Kim JH, Cheon JH, Kim WH. The frequency and the course of the adverse effects of azathioprine/6-mercaptopurine treatment in patients with inflammatory bowel disease. Korean J Gastroenterol 2008;51:291-297.

2. Khanna R, Feagan BG. Safety of infliximab for the treatment of inflammatory bowel disease: current understanding of the potential for serious adverse events. Expert Opin Drug Saf 2015;14:987-997.

3. Skef W, Hamilton MJ, Arayssi T. Gastrointestinal Behçet's disease: a review. World J Gastroenterol 2015;21:3801-3812.

4. Cheon JH, Han DS, Park JY, et al. Development, validation, and responsiveness of a novel disease activity index for intestinal Behçet's disease. Inflamm Bowel Dis 2011;17:605-613.

5. Kozarek RA, Patterson DJ, Gelfand MD, Botoman VA, Ball TJ, Wilske KR. Methotrexate induces clinical and histologic remission in patients with refractory inflammatory bowel disease. Ann Intern Med 1989;110:353-356.

6. Ardizzone S, Bollani S, Manzionna G, Imbesi V, Colombo E, Bianchi Porro G. Comparison between methotrexate and azathioprine in the treatment of chronic active Crohn's disease: a randomised, investigator-blind study. Dig Liver Dis 2003;35:619627.

7. Maté-Jiménez J, Hermida C, Cantero-Perona J, Moreno-Otero R. 6-Mercaptopurine or methotrexate added to prednisone induces and maintains remission in steroid-dependent inflammatory bowel disease. Eur J Gastroenterol Hepatol 2000;12:1227-1233.

8. Cheon JH, Kim WH. An update on the diagnosis, treatment, and prognosis of intestinal Behçet's disease. Curr Opin Rheumatol 2015;27:24-31.

9. Feagan BG, McDonald JW, Panaccione R, et al. Methotrexate in combination with infliximab is no more effective than infliximab alone in patients with Crohn's disease. Gastroenterology 2014;146:681-688.e1. 